\title{
Are genetic variations the most important risk factors for development of hepatocellular carcinoma?
}

\author{
Masafumi Ono $^{1} \cdot$ Toshiji Saibara $^{1}$
}

Received: 29 September 2015/Accepted: 30 September 2015/Published online: 31 October 2015

(C) Japanese Society of Gastroenterology 2015

In patients with type 2 diabetes mellitus (T2DM), cancer is reportedly the second most common cause of mortality, and hepatocellular carcinoma (HCC) is the most frequent type of cancer [1]. However, the pathogenetic factors for $\mathrm{HCC}$ development in patients with T2DM remain incompletely understood. Hyperinsulinemia in patients with T2DM is an important pathogenetic factor for the development of cancers, including HCC [2], because insulin promotes carcinogenesis and cell proliferation [2, 3]. Studies have shown that treatment with sulfonylurea and insulin is associated with a significantly increased risk, and that metformin is associated with a significantly decreased risk for the development of HCC in patients with T2DM [4, 5].

It has recently been clarified that an individual's genetic background is an important risk factor for the development of $\mathrm{HCC}$, even in patients with chronic liver diseases such as chronic hepatitis $\mathrm{C}(\mathrm{CH}-\mathrm{C})$, chronic hepatitis B (CH-B), alcoholic liver disease (ALD), and nonalcoholic fatty liver disease (NAFLD). The single-nucleotide polymorphism (SNP) located near interleukin 28B (IL28B) is reportedly related to development of $\mathrm{HCC}$ in patients with $\mathrm{CH}-\mathrm{C}$ [6]. Genetic variation of IL28B is a strong predictor of virological response to pegylated interferon- $\alpha$ and ribavirin combination therapy for $\mathrm{CH}-\mathrm{C}$. Asahina et al. [6] concluded that the cumulative incidence of HCC was significantly higher in the IL28B gene SNP among the patients who did not attain a sustained virologic response on $\mathrm{HCV}$

This comment refers to the article available at doi:10.1007/s00535015-1116-6.

Masafumi Ono

onom@kochi-u.ac.jp

1 Department of Gastroenterology and Hepatology, Kochi Medical School, Kochi, Japan genotype 1. Genetic variation of telomere maintenance genes (TMGs) is closely related to the development of $\mathrm{HCC}$ in patients with CH-B [7]. TMGs are essential for maintaining genomic integrity and stability by regulating the length of telomeres and the higher-order structure of protein complexes at the ends of chromosomes [8]. Among five TMGs, the telomerase-associated protein 1 rs 1713449 SNP was identified as the most significant risk factor for HCC development and overall survival in patients with $\mathrm{CH}-\mathrm{B}$. Moreover, genetic variation of the neurocan (NCAN) gene is a risk factor for $\mathrm{HCC}$ in patients with ALD. NCAN is a chondroitin sulfate proteoglycan within the extracellular matrix; it is expressed in neuronal tissue and is involved in brain development and remodeling [9]. Additionally, NCAN is a risk factor for the development of NAFLD, because it is also expressed in the liver [10]. Nischalke et al. [11] reported that the NCAN rs2228603 T-allele is an important risk factor for the development of $\mathrm{HCC}$ in patients with ALD.

Variation of the PNPLA3 gene (G-allele rs738409) is the most important genetic risk factor for the development and severity of NAFLD according to genome-wide association studies $[12,13]$. The G-allele of the PNPLA3 gene is closely associated with Matteoni type 4 nonalcoholic steatohepatitis (NASH) in the Japanese population [14]. With respect to the relationship between $\mathrm{HCC}$ and the PNPLA3 gene, the PNPLA3 G-allele is associated with an increased risk of advanced fibrosis and is an independent risk factor for the development of HCC among patients with NASH or ALD-related cirrhosis [15, 16].

Ueyama et al. [17] first reported that genetic variation of PNPLA3 was associated with HCC development in patients with T2DM. Although T2DM is an important risk factor for $\mathrm{HCC}$, the associated genetic background remains unclear. Moreover, it has been considered that HCC 
frequently develops in association with advanced NASH in patients with T2DM because patients with T2DM frequently have NASH and NAFLD. Because the proportion of fatty liver disease in each group was not different in this study, however, the development of HCC might not always be related to the presence of NASH. However, the higher proportion of liver cirrhosis and relatively lower proportion of fatty liver in the DM-HCC group may have been related to the presence of "burned-out NASH." Although further detailed examination including histological evaluation is needed, it is notable that both liver cirrhosis and PNPLA3 rs738409 G-allele were independent risk factors for the development of HCC. Furthermore, the authors were the first to identify another important genetic risk factor, genetic variation of JAZF1, for the development of HCC in patients with T2DM who are homozygous for the PNPLA3 $\mathrm{G}$-allele. The JAZF1 gene plays a role in regulation of lipid metabolism [18]. Genetic variation of the JAZF1 gene is reportedly closely associated with susceptibility to T2DM and traits of insulin secretion [19]. Additionally, colon and prostate cancer are also reportedly related to the $\mathrm{T}$-allele of the JAZF1 gene [20]. Ueyama et al. [17] concluded that the combination of the PNPLA3 G-allele and JAZF1 G-allele may be a more important predictive factor for the development of HCC.

Accordingly, this study provides beneficial information regarding the mechanisms of genetic variation (PNPLA3 and JAZF1) for development of HCC in patients with T2DM. Further investigations are expected to clarify the details regarding genetic variations and functions of the genes in HCC development.

\section{References}

1. Emerging Risk Factors Collaboration, Seshasai SR, Kaptoge S, Thompson A, et al. Diabetes mellitus, fasting glucose, and risk of cause-specific death. N Engl J Med. 2011;364:829-41.

2. Kawaguchi T, Izumi N, Charlton MR, et al. Branched-chain amino acids as pharmacological nutrients in chronic liver disease. Hepatology. 2011;54:1063-70.

3. Barker BE, Fanger H, Farnes P. Human mammary slices in organ culture. I. method of culture and preliminary observations on the effect of insulin. Exp Cell Res. 1964;35:437-48.

4. Singh S, Singh PP, Singh AG, et al. Anti-diabetic medications and the risk of hepatocellular cancer: a systematic review and meta-analysis. Am J Gastroenterol. 2013;108:881-91.
5. Kawaguchi T, Kohjima M, Ichikawa T, et al. The morbidity and associated risk factors of cancer in chronic liver disease patients with diabetes mellitus: a multicenter field survey. J Gastroenterol. 2015;50:333-41.

6. Asahina Y, Tsuchiya K, Nishimura T, et al. Genetic variation near interleukin $28 \mathrm{~B}$ and the risk of hepatocellular carcinoma in patients with chronic hepatitis C. J Gastroenterol. 2014;49: 1152-62.

7. Jung SW, Park NH, Shin JW, et al. Prognostic impact of telomere maintenance gene polymorphisms on hepatocellular carcinoma patients with chronic hepatitis B. Hepatology. 2014;59:1912-20.

8. Blasco MA. Telomeres and human disease: ageing, cancer and beyond. Nat Rev Genet. 2005;6:611-22.

9. Rauch U, Karthikeyan L, Maurel P, et al. Cloning and primary structure of neurocan, a developmentally regulated, aggregating chondroitin sulfate proteoglycan of brain. J Biol Chem. 1992;267:19536-47.

10. Palmer ND, Musani SK, Yerges-Armstrong LM, et al. Characterization of European ancestry nonalcoholic fatty liver diseaseassociated variants in individuals of African and Hispanic descent. Hepatology. 2013;58:966-75.

11. Nischalke HD, Lutz P, Krämer B, et al. A common polymorphism in the NCAN gene is associated with hepatocellular carcinoma in alcoholic liver disease. J Hepatol. 2014;61:1073-9.

12. Romeo S, Kozlitina J, Xing C, et al. Genetic variation in PNPLA3 confers susceptibility to nonalcoholic fatty liver disease. Nat Genet. 2008;40:1461-5.

13. Rotman Y, Koh C, Zmuda JM, NASH CRN, et al. The association of genetic variability in patatin-like phospholipase domaincontaining protein 3 (PNPLA3) with histological severity of nonalcoholic fatty liver disease. Hepatology. 2010;52:894-903.

14. Kawaguchi T, Sumida Y, Umemura A, Japan Study Group of nonalcoholic fatty liver disease, et al. Genetic polymorphisms of the human PNPLA3 gene are strongly associated with severity of non-alcoholic fatty liver disease in Japanese. PLoS One. 2012;7:e38322.

15. Takeuchi Y, Ikeda F, Moritou Y, et al. The impact of patatin-like phospholipase domain-containing protein 3 polymorphism on hepatocellular carcinoma prognosis. J Gastroenterol. 2013;48:405-12.

16. Liu YL, Patman GL, Leathart JB, et al. Carriage of the PNPLA3 rs738409 C > G polymorphism confers an increased risk of nonalcoholic fatty liver disease associated hepatocellular carcinoma. J Hepatol. 2014;61:75-81.

17. Ueyama M, Nishida N, Korenaga M, et al. The impact of PNPLA3 and JAZF1 on hepatocellular carcinoma in non-viral hepatitis patients with type 2 diabetes mellitus. J Gastroenterol. 2015;. doi:10.1007/s00535-015-1116-618.

18. Li L, Yang Y, Yang G, et al. The role of JAZF1 on lipid metabolism and related genes in vitro. Metabolism. 2011;60:523-30.

19. Omori S, Tanaka Y, Horikoshi M, et al. Replication study for the association of new meta-analysis-derived risk loci with susceptibility to type 2 diabetes in 6244 Japanese individuals. Diabetologia. 2009;52:1554-60.

20. Thomas G, Jacobs KB, Yeager M, et al. Multiple loci identified in a genome-wide association study of prostate cancer. Nat Genet. 2008;40:310-5. 